\title{
A New Behavior of Nuclei during Mitosis of Lilium Hybrids
}

Sixiang Zheng ${ }^{1}$, Feihu Liu ${ }^{2}$, Chaojun Zhang ${ }^{1}$, Zhilin Chen $^{1}$, Yuhua $\mathrm{Ma}^{1}$, Pinghong

Meng ${ }^{1}$, Yan Zhao ${ }^{3}$

1 Horticultural Research Institute, Guizhou Academy of Agricultural Science, Guiyang 550006, China

2 Faculty of Life Science, Yunnan University, Kunming 650091, China

3 The Gardening Department of Horticulture College, Yunnan Agriculture University, Kunming 650201, China

e-mail:zhengsixiang1966@126.com

Mitosis is nuclear division plus cytokinesis,and produces two identical daughter cells during prophase,prometaphase,metaphase,anaphase, and telophase.Mitosis occurs exclusively in eukaryotic cells, but occurs in different ways in different species. For example, animals undergo an "open" mitosis, where the nuclear envelope breaks down before the chromosomes separate, while fungi such as Aspergillus nidulans and

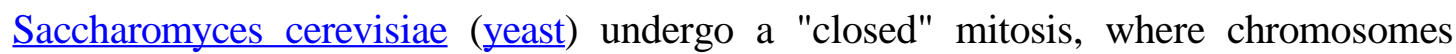
divide within an intact cell nucleus ${ }^{1}$. Prokaryotic cells, which lack a nucleus, divide by a process called binary fission ${ }^{2}$.

However, an unusual nucleus behavior in interspecific hybrid progenies of Lilium was observed in our experiment. Lily (Lilium spp.) is one of the most important ornamental bulb crops worldwide. It is widely cultivated as cut flower, pot and garden plant. Lilies of ornamental importance are the result of crosses within different sections and interspecific hybrids within sections have been bred and cultivated widely since 
early 1800 s . Using the methods ${ }^{3}$ of in vitro fertilization and embryo rescue, interspecific hybrid lily was obtained from Lilium Oriental 'Ruyi'(O) x L.sulphureum(S)(OS hybrids). Further crosses were made utilizing one OS lily x 'Siberia', OT lily 'Manissa' $\mathrm{x}$ 'Siberia' ${ }^{4}$.Root tips of hybrid progenies from $\mathrm{OT} \times \mathrm{O}$ and $\mathrm{OS} \times \mathrm{O}$ of Lilium were directly fixed in Carnoy solution (ethanol: acetic acid $=3: 1$ ) for $12 \mathrm{~h}$ and digested for 10 min in $0.1 \mathrm{M} \mathrm{HCl}$. Root tips were rinsed for $10 \mathrm{~min}$ in distilled water. The root tips were mounted on a glass slide with a fresh drop of $10 \%$ modified phenol-fuchsin solution and covered with a cover slip. The glass slide was warmed by alcohol flame for a few seconds. The cover slip was tapped gently with fine forceps and squashed using the thumb. Nucleus division phases were examined under an Olympus microscope. Very unusual behaviors of nuclei surprisingly presented, such as sprouting or germination(Fig.1a), tube-like elongation(Fig.1b), penetrating cell membrane into a neighbor cell(Fig.1e), the top of nuclei tube expanding(Fig.1c), intruding and splitting of the tube-like nucleus(Fig.1d), and micronucleus formation(Fig.1f), and so on. Furthermore, the tetrad of meiosis was founded in mitosis of $\operatorname{root}($ Fig.1d).

Routine of the unusual nucleus behaviors observed in our experiment may be summarized as nucleus germination - tube-like elongation- penetrating cell membrane- entering a neighbor cell-the top of nuclei tube expanding-tube ingression and splitting — formation of a new nucleus or micronucleus.

Many kinds of abnormal mitosis caused by chemical and physical induction such as unequal division, chromosome bridges, lagging chromosomes, and multiple nuclei have resulted in variations of chromosome number and structure ${ }^{5,6,7}$. However, this new 
nucleus behavior is firstly reported, these phenomena implied that the DNA maybe easily emigrates from one cell to another. Therefore, the unusual behaviors of nuclei in hybrid progenies of Lilium not only create mutations for breeding of new cultivars, also produce possibly ideal materials for exotic DNA or gene transfication with simple method in meristem. This mode of nuclei behaviors is a new addition to cytogenetics of plant of vegetative propagation and provide a new genetic mechanism of species evolution from interspecific hybridization.

1.De S. C.P.\&Osmani, S.A.Eukaryotic Cell 6, 1521-7(2007)..

2.Nanninga N. Microbiol Mol Biol Rev 65 , 319-333 (2001).

3.Van Tuyl, J.M., Van Die“n, M.P.\&Van Creij, M.G.M. Plant Sci. 74, 115-126(1991).

4.Zheng, S.-X. Wang, J.-H.\&Hu ,C. Journal of West China Forestry Science 38 ,5-8(2009).

5. Wu, H.Z. Zheng, S.X.\& He, Y.-Q. Scientia Horticulturae 114, 50-53(2007).

6. Xu, Q.-M., Cheng, J-S.\& Ge, Z.-Q. Enzyme and Microbial Technology 37, 7681(2005).

7.Zheng, S.-X., Zhang, F.-Q.\&Li, Z.-D. Acta Agronomica Sinica,26, 347-351(2000). 

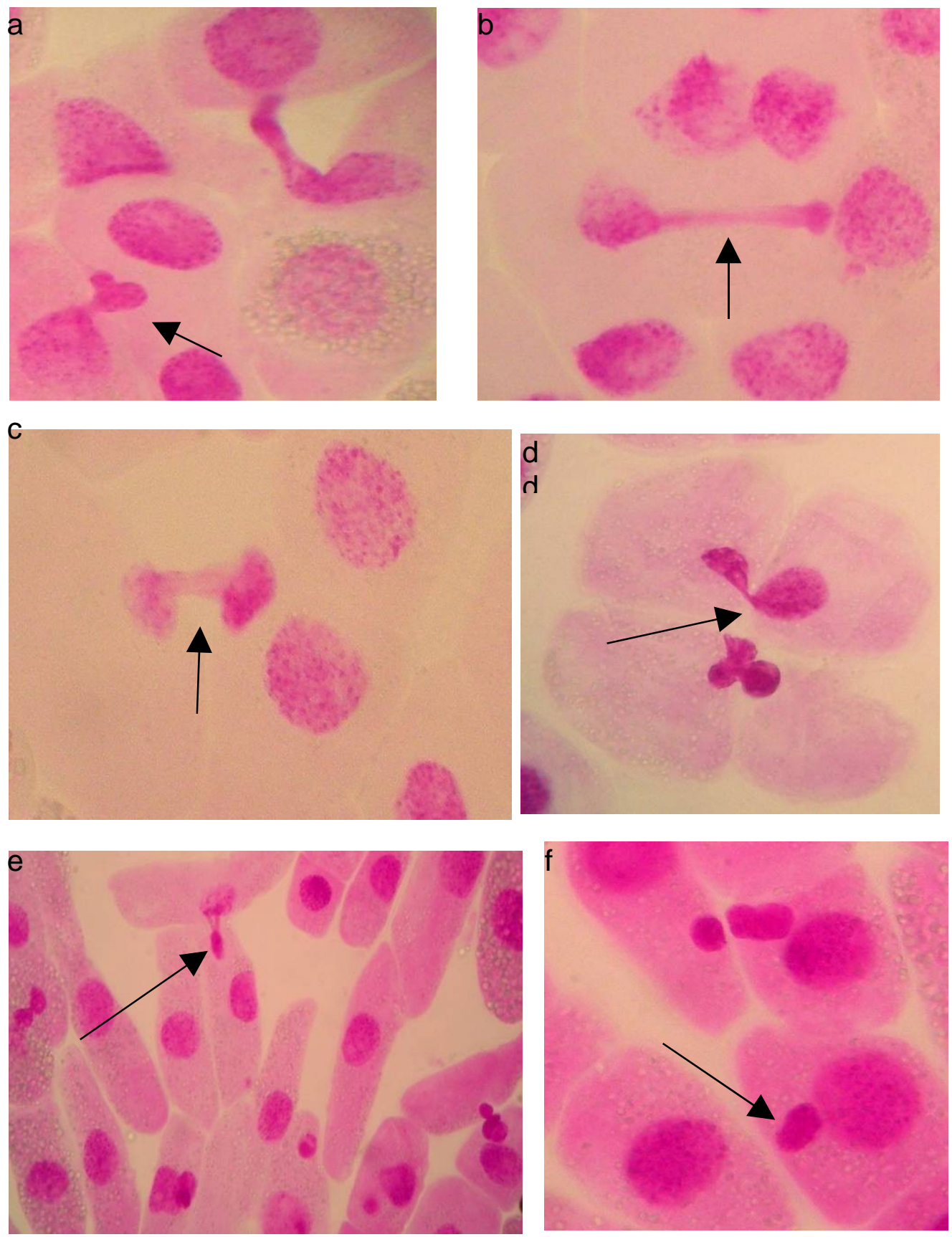

Fig 1 Abnromal mitosis and new behaviors of nuclei $(\mathrm{OT} \times \mathrm{O}, \mathrm{OS} \times \mathrm{O})$

a. Nucleus sprouting; b. Nucleus tube-like elongation; c. The top of nuclei tube expanding; d. Ingression and splitting of tube-like nucleus; e. Penetrating cell membrane and entering the neighbor cell; f. Micronucleus formation 